\title{
Patient engagement in clinical guidelines development: input from > 1000 members of the Canadian Osteoporosis Patient Network
}

\author{
S. N. Morin ${ }^{1,2}$ (D) M. Djekic-Ivankovic ${ }^{2} \cdot$ L. Funnell ${ }^{3} \cdot$ L. Giangregorio $^{4} \cdot$ I. B. Rodrigues ${ }^{4} \cdot$ R. Ridout ${ }^{5} \cdot$ S. Feldman ${ }^{5}$. \\ S. Kim ${ }^{5} \cdot$ H. McDonald-Blumer ${ }^{5}$. G. Kline ${ }^{6}$-W. E. Ward ${ }^{7}$ - N. Santesso ${ }^{8}$ - W. D. Leslie ${ }^{9}$
}

Received: 12 October 2019 / Accepted: 22 November 2019 / Published online: 14 December 2019

(C) The Author(s) 2019

\begin{abstract}
Summary Patient engagement in clinical guidelines development is essential. The results of a self-administered online survey identified themes important to people living with osteoporosis and will inform the development of Osteoporosis Canada clinical guidelines recommendations.

Introduction Patient engagement is essential in the development of high-quality and relevant guidelines for osteoporosis management. Osteoporosis Canada (OC) is updating its national clinical practice guidelines in collaboration with people living with osteoporosis in the process.

Methods Using electronic mail, we contacted 6937 members of the Canadian Osteoporosis Patient Network (COPN) to provide input on the selection of relevant content, outcomes, and research questions via a self-administered online survey. Close-ended questions were analyzed using descriptive statistics, and conventional content analysis was conducted for open-ended questions. Results A total of 1108 individuals completed the survey (97\% women, 86\% stated they lived with osteoporosis). Most participants considered it critical to have recommendations on physical activity and exercise (74\%), fall prevention (69\%), nutrition $(68 \%)$, and initial bone mineral density testing (67\%). In addition to preventing fractures, over $75 \%$ of respondents stated that consideration of preserving quality of life and ability to perform daily activities, preventing admission to long-term care and fracture-related death, and avoiding serious harms from medications were essential outcomes to consider in evaluating the evidence. In terms of selection of research questions, seven themes emerged from the content analysis including pharmacotherapy, screening and monitoring, diet and supplements, education, exercise, alternative therapies, and pain management.

Conclusions Patients emphasized that autonomy, mobility, and quality of life are highly valued outcomes and must be integral to practice guideline development. As expected, guidance on pharmacotherapy, screening and monitoring, and fracture prevention were priorities identified to be included in osteoporosis management guidelines.
\end{abstract}

Keywords Clinical guidelines · Osteoporosis · Patient engagement · Survey

Electronic supplementary material The online version of this article (https://doi.org/10.1007/s00198-019-05248-4) contains supplementary material, which is available to authorized users.

\section{S. N. Morin}

suzanne.morin@mcgill.ca

McGill University, Montreal, Canada

2 Centre for Outcomes Research and Evaluation, Research Institute of the McGill University Health Centre, 5252 de Maisonneuve O, Room 3E.11, Montreal, Quebec H4A 3S5, Canada

3 Osteoporosis Canada, Toronto, Canada
4 University of Waterloo and Schlegel-UW Research Institute for Aging, Waterloo, Canada

5 University of Toronto, Toronto, Canada

6 University of Calgary, Calgary, Canada

Brock University, St. Catharines, Canada

8 McMaster University, Hamilton, Canada

9 University of Manitoba, Winnipeg, Canada 


\section{Introduction}

Osteoporosis is a chronic disease characterized by low bone mass and deterioration of bone tissue associated with an increased risk for fracture [1]. People living with osteoporosis face reduced quality of life and loss of mobility and autonomy [2]. Worldwide, at least one in three women and one in five men over the age of 50 years will sustain an osteoporotic fracture during their lifetime. Clinical practice guidelines have been developed in most jurisdictions and provide countryspecific frameworks for osteoporosis management and fracture prevention to clinicians, patients, and healthcare administrators $[3,4]$.

Patient engagement is essential for the development of high-quality and relevant clinical practice guidelines. Scientific and patient organizations and research funding bodies have urged greater patient participation in knowledge synthesis and research activities, emphasizing the important stake that patients have in the outcomes and endorsing patients' unique experiences and valuable perspectives. The Guidelines International Network and the National Academy of Medicine recommend that patients or patient representatives be engaged on guideline development panels alongside physicians, researchers, and other professionals [5, 6]. Patient participation influences the inclusion of patient-relevant topics, outcomes selection, and approaches to recommendation development [7].

Nevertheless, patient and public involvement in guideline development remains suboptimal [8]. There are challenges to engaging patients in research and guideline development including recruiting patients, inadequate training and support, difficulties in understanding medical terminology, lack of resources for patients' engagement, and uncertainty of how to incorporate patient experiences into evidence-based guidelines $[7,9,10]$. To overcome these barriers, strategies such as involving trained professionals for recruitment, clear communication, clarity regarding the patient role, and early engagement of patients throughout the entire guideline process have been proposed [11].

Osteoporosis Canada is currently updating its national clinical practice guidelines using the GRADE framework [12] and has been successful in assuring active patient collaboration throughout the entire process. Founded in 2004 by four friends who were determined to overcome the challenges of living with osteoporosis, the Canadian Osteoporosis Patient Network (COPN) is the patient arm of Osteoporosis Canada (OC). Within OC, COPN has an organizational structure, receives OC staff support, and participates actively in knowledge transfer activities, in white paper development and as an advisory body to national research funding bodies. Some of its members are actively involved as patient-partners in peerreviewed research and quality of care improvement programs. COPN connects individuals affected by osteoporosis, communicates information about how to live well with the disease, and advocates for comprehensive access to diagnosis, treatment, and care. Its membership has recently reached 10,000 individuals across the country (https://osteoporosis. ca/bone-health-osteoporosis/living-with-the-disease/copn/).

As part of OC guideline update process, COPN members are active and engaged participants of the steering, conflict of interest and knowledge transfer committees, and of each of 4 working groups tasked with the development of key recommendation questions and systematic reviews [13]. To inform the search strategy for knowledge syntheses and the eventual development of the clinical recommendations, COPN members have participated in the development, distribution, and evaluation of an electronic survey of their national membership with the primary objective to identify issues important to people living with osteoporosis in the development of the clinical guidelines. We report the survey results which presently informs the $\mathrm{OC}$ osteoporosis management guidelines update process.

\section{Methods}

\section{Study population}

A self-administered online survey was developed and distributed using electronic mail to 6937 COPN members with email access from April 17 to May 8, 2018. COPN members were notified twice of the survey's imminent release through the distribution of an e-newsletter. The survey was distributed in a separate e-blast to ensure its visibility. A reminder to complete the survey was sent 2 weeks after the initial invitation. The survey was posted on SurveyMonkey ${ }^{\mathrm{TM}}$ (SurveyMonkey Inc., Palo Alto, CA, USA), an online survey development cloud-based software, is compliant with Canadian privacy and accessibility standards (W3C), and does not collect computer internet protocol (IP) address or any other participant identifying information. Consent to participate was assumed if the survey was completed and submitted. The study was approved by the University of Manitoba Health Research Ethics Board.

\section{Survey design}

The survey was developed by the OC osteoporosis guideline steering committee including clinicians, patients, and researchers with expertise in bone health as well as in survey and guidelines development. Questions were developed to engage participants by using specific web-based survey design, structure, and technical interface [14] and were designed to acquire input about the future guidelines' content and selection of research questions. There were 10 close-ended questions and 2 open-ended questions. Three questions 
pertained to limited demographic information and to knowledge about osteoporosis (very little knowledge, a little knowledge, neutral, somewhat knowledgeable, very knowledgeable), three questions inquired about the importance of including guidance on specific recommendations and clinical outcomes (1-2, not important to consider; 3-4, important; 5, critical to consider), two questions focused on how to get information about fracture risk assessment and healthcare provider visit, and two questions were on exercise and medication and had a free text box to allow for additional comments (Supplemental Appendix- survey). Participants were asked for inputs and comments through 2 open-ended questions: (1) Provide up to three specific questions that you would like to see addressed in the next Canadian Osteoporosis Clinical Practice Guidelines and (2) What questions do you have about the safety or effectiveness of exercise, or safe performance of physical activities of leisure or daily living. Clarity, content, and interface validity of the survey were verified through pilot testing with 53 participants. Recommended changes were incorporated into the final survey prior to distribution. The survey was translated into French for distribution to COPN French-speaking members.

\section{Analysis}

Data were analyzed using descriptive statistics for close-ended questions with results expressed as frequencies and percentages.

Content analysis was conducted for the open-ended questions using NVivo software version 11 by QSR International Ltd. $[15,16]$. Initial step of content analyses consisted of preliminary reading of raw data to recognize respondents' perceptions. Primary coding level of raw data was conducted for each question by identifying every idea. Identified ideas were coded by simple induction [17] without predetermined themes or research-based expectations [18]. Second level of coding was focused on grouping the ideas to create categories described as inductive analyses of codes to create categories or subthemes. Finally, thematic coding as a third level of analyses studied previous coding to develop highly refined themes representing a group of categories of ideas. Two independent researchers coded the data using described qualitative analyses methodology based on content analyses. Figures for closeended questions were generated using GraphPad Prism ver. 6.0 (La Jolla, CA, USA).

\section{Results}

A total of 1108 completed surveys were obtained (16\% participation rate from 6937 members with electronic email addresses). Most respondents were women $(96 \%, N=1059)$ and $85 \%$ reported living with osteoporosis, $84 \%$ of the men respondents also reported having osteoporosis; $4.8 \%$ claimed to be an allied health professional and $0.6 \%$ to be a physician. Sixty-one percent of respondents had some knowledge about osteoporosis while $24 \%$ reported to be very knowledgeable and $1.3 \%$ to have very little knowledge. The majority of participants were from the provinces of Ontario $(N=534)$ and British Columbia $(N=186)$, with less than 100 participants from each of the other provinces and territories. Not all participants answered all questions (range 10 to 123 responses missing per question) (Table 1).

Most participants considered it critical (5 on a 5-point rating scale) to have recommendations on physical activity and exercise (74\%), fall prevention (69\%), nutrition (68\%), initial bone mineral density testing $(67 \%)$, and management of acute pain from fractures $(66 \%)$ (Fig. 1a). The weighted averages for responses for these recommendations were all above 4.5, with less than $2 \%$ choosing the category of "not important to consider".

Over $75 \%$ of respondents stated that preserving quality of life and well-being (87\%) and ability to perform daily physical and social activities (84\%), preventing admission to long-term care $(84 \%)$ and fracture-related death $(85 \%)$, preventing all fractures (79\%), and avoiding serious harms from medications (78\%) were critical outcomes to consider in guidelines development (Fig. 1b). The weighted averages for responses for these recommendations were all above 4.7 , with less than $1.5 \%$ choosing the category of "not important to consider".

Discussion with a physician (average 4.2) or with another healthcare professional (average 4.1), or access to material posted on the OC website (average 4.1), was determined to

Table 1 Survey participants' characteristics

\begin{tabular}{ll}
\hline Participants' characteristics & $N=1108$ \\
\hline Sex, $n(\%)$ & $1059(96 \%)$ \\
$\quad$ Women & \\
Language, $n(\%)$ & $1036(94 \%)$ \\
$\quad$ English & \\
Osteoporosis, $n(\%)$ & $943(86 \%)$ \\
I have OP & $29(3 \%)$ \\
I care for someone with OP & \\
Place of residence of participants in Canada $(\%)$ & $34 \%$ \\
Western Canada & $58 \%$ \\
Central Canada & $8 \%$ \\
Atlantic Canada & $<1 \%$ \\
Northern Canada & \\
Osteoporosis knowledge (\%) & $85 \%$ \\
Knowledgeable $^{1}$ & $15 \%$ \\
Not knowledgeable &
\end{tabular}

${ }^{1}$ Knowledgeable includes very knowledgeable and knowledgeable; ${ }^{2}$ Not knowledgeable includes little knowledgeable, very little knowledgeable, or neutral 
a) Recommendations critical to consider including in osteoporosis guideline development

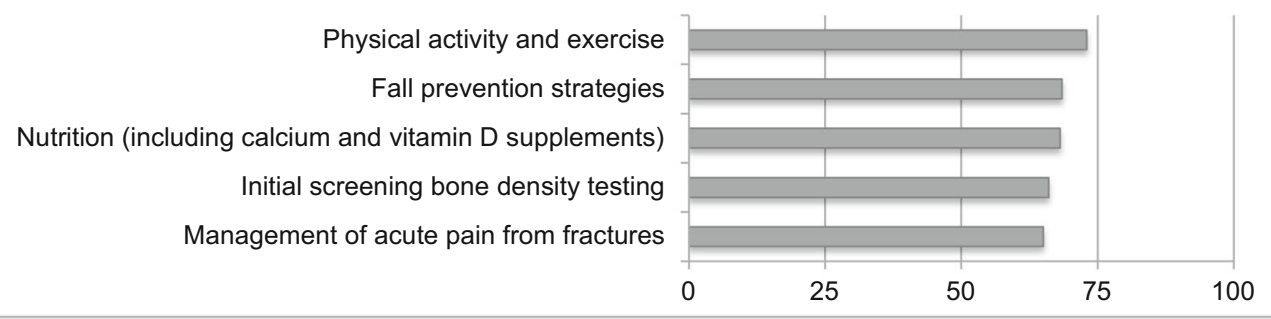

b) Outcomes critical to consider in osteoporosis management guideline development

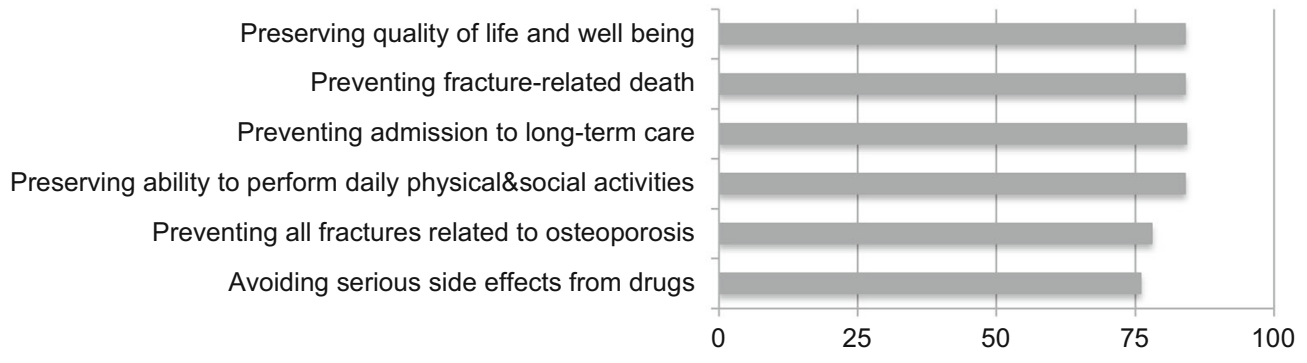

Fig. 1 Percentage of respondents who indicated that specific recommendations (a) and outcomes (b) were critical to consider in the updated osteoporosis clinical guideline development

be critical in ensuring a good understanding of the risks and consequences of fractures by $51 \%, 41 \%$, and $41 \%$ of the respondents respectively. The majority of participants $(64 \%)$ would be willing to spend $15 \mathrm{~min}$ to $1 \mathrm{~h}$ preparing themselves for a visit and discussion with their physician on fracture risk and $55 \%$ felt they would likely require a discussion of more than 10 min with their physician to understand the result of their fracture risk assessment.

Results of the close-ended question on exercise (why do you choose to participate in exercise? enter up to 5 answers) revealed that most respondents chose to exercise for its effect on balance (77\%), strength (64\%), mobility (53\%), and quality of life $(51 \%)$. Other benefits were also noted to be of importance: effect on posture $(45 \%)$, on preservation of BMD (42\%), on endurance (43\%), on risk of falls or broken bones $(42 \%)$, and on the ability to do daily exercises (41\%). The effect of exercise on mood was felt to be important only for a minority $(11 \%)$. In addition to those pre-specified answers in the exercise close-ended question, $7 \%$ of participants provided comments in the "Other" answer box generating 63 references. Key issues identified included benefits of exercise on general health (19 references: autonomy, strength, balance and flexibility, social interactions, well-being, and pain reduction), benefits of exercise on the skeleton (16 references: safety of exercises/movements, what exercises improve BMD, and optimal exercise frequency), and barriers to exercising (20 references: lack of access to facilities/trained professionals, perceived difficulty due to pain, lack of time, negative attitudes towards exercise, and lack of guidance by primary care professionals).
We specifically inquired whether participants had specific questions about the safety or effectiveness of exercise in an open-ended question (Table 2). Three hundred sixty-eight participants provided 586 references, most pertained to what exercises or types of exercise (e.g., yoga) were safe versus what to avoid $(n=222)$ and what type or dose of exercises was effective $(n=145)$. There was significant interest in how to access safe and effective exercises (e.g., online videos, community groups, trained professionals; $n=116$ ). In addition, participants were interested to know more about the benefits of exercise on bone mineral density, fracture risk, balance and strength, as well as pain and quality of life.

We asked participants to select up to 5 items from a list of questions or issues related to medications for osteoporosis they would like addressed in the updated guidelines to help guide healthcare professionals in making treatment decisions with their patients. The most frequently noted items were as follows: how to choose the best medication option (81\%), how to describe the risks and benefits of each medication (81\%), how long a medication should be taken $(67 \%)$, how to tell if a medication is working (65\%), and how to watch for side effects including common and rare events (65\%). A free text box allowed respondents to provide additional input leading to 135 references (Table 3). Most responses were related to pharmacological treatment ( 75 references) with $73 \%$ of references related to benefits and harms of the medications. Furthermore, there was interest in ensuring that guidance was provided to healthcare professionals about nutritional supplements $(n=19)$ and alternative therapies (e.g., natural products, naturopathy; $n=14$ ). 
Table 2 Participants' responses to the open-ended question: What questions do you have about the safety or effectiveness of exercise, or safe performance of physical activities of leisure or daily living?

\begin{tabular}{ll}
\hline Number of participants who provided answers & $\begin{array}{l}N=368 \\
\text { Total references* }\end{array}$ \\
& 586 \\
\hline How can I exercise safely? & 222 \\
Safety of specific/preferred exercises (yoga etc.) & 69 \\
What exercises to avoid & 56 \\
What exercises/classes/movements are safe to do & 53 \\
Concerns about adverse effects (including pain) & 31 \\
If I have a vertebral fracture & 14 \\
How can I exercise effectively? & 145 \\
Best/most effective exercises for my condition & 88 \\
Right frequency/duration/intensity of exercise/strength training & 57 \\
How can I access safe and effective exercises? & 116 \\
Trained professionals & 48 \\
Make adapted exercises available online/on video/at home & 35 \\
Community/support groups/age-specific classes & 33 \\
What are the benefits of exercise on: & 72 \\
Bone mineral density & 23 \\
Fracture risk & 23 \\
Balance and strength & 15 \\
Pain and quality of life improvement & 11 \\
Other & 31 \\
\hline
\end{tabular}

*Each participant could give more than one idea: 1 idea $=1$ reference. Total indicates the number of references among the participants who answered the open-ended question
Table 4 summarizes results of content analyses for suggestions of research questions for consideration for evidence synthesis ("Provide up to three specific questions you would like to see addressed in the next Canadian Osteoporosis Clinical Practice Guidelines"). Participants provided 964 references that emerged into 8 main themes that we tabulated in descending order of importance: pharmacological treatment (e.g., benefits and harms, new medications, duration of therapy, drug holiday); screening, monitoring, and prevention (BMD testing); nutrition and supplements; exercise and physical activity; knowledge, education, and advocacy; alternative therapies; and pain. One-third of the participants' responses (327 references) were related to pharmacological treatments including benefits and harms, new medications or the best medication, duration of therapy, as well as drug holiday.

Finally, we separately analyzed the answers provided by the men respondents and found no differences when compared with those provided by women respondents.

\section{Discussion}

We engaged patients as partners in priority setting to inform the development of national osteoporosis guidelines and learned that recommendations on pharmacotherapy, screening and monitoring, nutrition, and exercise were fundamental in the upcoming guidelines. However, in addition to fracture prevention, over $75 \%$ of participants responded that outcomes such as preservation of autonomy, mobility, and quality of life were essential to consider when developing recommendations for osteoporosis management.

Mobility can be seriously diminished in patients with osteoporosis, particularly in those who have sustained fractures [19]. Kerr et al. have also documented, through patient and clinician interviews, the importance of the impact of osteoporosis on physical function [20]. They described a cycle of impairment characterized by the interaction of numerous factors such that limitations in activities lead to loss of muscle and bone strength with a subsequent increase in fracture risk, which in turn will result in pain and fear of falling and further limitations in the performance of daily activities. This accumulation of physical impairments and fear can precipitate institutionalization and dependence on others for simple tasks. Our findings were similar in that mobility and autonomy were highly valued by respondents of this survey.

Participants identified physicians and healthcare professionals as their preferred source of information on osteoporosis and fractures and they are agreeable to spending a significant amount of time to prepare for a physician visit and to discuss the results of fracture risk assessment. Therefore, in addition to guideline development, we will engage in the development and implementation of tools to support 
Table 3 Participants' responses to the open-ended question: What questions or issues related to medications for osteoporosis would you like to see addressed in the next Canadian Osteoporosis Clinical Practice Guidelines in order to help doctors and other healthcare professionals in making treatment decisions with their patients?

\begin{tabular}{ll}
\hline Number of participants who provided answers & $N=113$ \\
& Total references* \\
& 135 \\
\hline Pharmacologic treatment & 75 \\
Benefits and harms & 55 \\
Cost of medications & 7 \\
Drug-drug interactions & 5 \\
New medications & 4 \\
Drug holiday & 2 \\
Access to medications & 2 \\
Time to start with a therapy & 1 \\
Nutrition and supplements & 1 \\
Supplements recommendations & 19 \\
Dietary recommendations & 12 \\
Alternative therapies & 7 \\
Education & 14 \\
For patients/families/caregivers-source of information they can trust & 11 \\
For healthcare professionals & 6 \\
Monitoring-prevention & 5 \\
Response to treatment/BMD & 5 \\
Prevention & 5 \\
Access to specialists & 5 \\
Pain management & 6 \\
Other & 1 \\
\hline & 1 \\
\hline & 6 \\
\hline
\end{tabular}

*Each participant could give more than one idea: 1 idea $=1$ reference. Total indicates the number of references among the participants who answered the open-ended question knowledge transfer and shared decision-making at the time of the guidelines publication.

Evidence of consideration for patient beliefs, values, and preferences is often not provided in osteoporosis clinical practice guidelines [21]. In a recent review of seventy international guidelines, only $39 \%$ included statements about patients' values and preferences; most of these statements were conceptualized as preferences for one medication over another or in terms of financial costs of therapies. To complicate matters, ascertainment and understanding of patient preferences can differ between patients and guideline developers such that, for example, one would consider options in terms of treatment (including no treatment) while the other would expect choices between various treatments [22]. Although respondents in our survey mentioned specific questions on pharmacotherapy with high frequency, a variety of other considerations such as exercise, diet, education and alternative therapies, and pain were also noted, expanding the field of interest outside of the traditional pharmacologic focus.

Accurate perception of risk is crucial for the rational adoption of treatment. Communicating risk and benefit of pharmacological treatment to patients remains a great challenge [23]. Many tools and strategies [24], developed by investigators (such as the Mayo Clinic Osteoporosis Choice decision aid https://osteoporosisdecisionaid.mayoclinic.org/index.php/ site/index) and patient societies, aim to support decisionmaking; however, in our experience and as has been highlighted in recent publications [25], healthcare providers' messages about fracture risk are confusing to patients and much work is still required so that communications better suit patients' needs.

The patient perspective is now recognized as fundamental in health research, guideline development, and treatment regulatory processes and recommended by many organizations [26]. Growing recognition that people with lived experience can make important contributions to the study of health and healthcare has resulted in increasing participation of patients in different levels of involvement from consultation such as surveys and interviews to active engagement like shaping research questions. OC is fortunate to count on its COPN members' involvement in the updating of the clinical guidelines and in many other research activities. Future research protocols may also benefit from this type of background data to inform study design and outcomes that are important to patients beyond simple measures of fracture occurrence. Our ongoing engagement will support the successful creation of relevant practice guidelines that incorporate patients' values and perspectives and encourage shared decision-making. 
Table 4 Participants' responses to the open-ended question: Provide up to three specific questions you would like to see addressed in the next Canadian Osteoporosis Clinical Practice Guidelines

\begin{tabular}{ll}
\hline Number of participants who provided answers & $N=498$ \\
& Total references* \\
& 964 \\
\hline Pharmacological treatment & 327 \\
Benefits and harms & 183 \\
New medications/the best medication & 58 \\
Duration of therapy/drug holiday & 54 \\
Other (cost, medication choice, etc.) & 32 \\
Screening-monitoring-prevention & 193 \\
BMD tests/response to treatment & 88 \\
Prevention & 65 \\
Early screening & 30 \\
Other (access to specialists, clinics, etc.) & 10 \\
Nutrition and supplements & 140 \\
Dietary recommendations & 59 \\
Supplements recommendations & 52 \\
Dietary sources of calcium & 22 \\
Other (vitamin K, protein) & 7 \\
Exercise-physical activity & 121 \\
Recommendations & 80 \\
Safety and benefits & 31 \\
Other (fit trainers, cost, etc.) & 10 \\
Knowledge-education-advocacy & 135 \\
Patients/families/caregivers & 72 \\
Healthcare professionals & 22 \\
Advocacy/support groups & 17 \\
General public & 13 \\
Other (list of specialists, funding, etc.) & 13 \\
Alternative therapies & 30 \\
Naturopathy/natural products & 28 \\
Other & 2 \\
Pain & 13 \\
Cain mannagement & \\
Other (osteoporosis pain vs. arthritis pain) & \\
\hline
\end{tabular}

*Each participant could give more than one idea: 1 idea $=1$ reference. Total indicates the number of references among the participants who answered the open-ended question

Our study is limited by the modest response rate and the fact that those who answered likely constitute a select group with a special interest in guideline development and engagement in such projects. Very few men participated in the survey. Still, over one thousand COPN members responded to the survey request, which is a very large number of respondents for a non-remunerated patient survey. Furthermore, the respondents' demographics are representative of the national COPN membership, supporting the robustness of our data.
Also, most respondents claimed to have at least some background knowledge of osteoporosis with many being very knowledgeable. It is therefore possible that lessengaged patients may be less willing to do pre-visit "homework" in preparation for a physician visit. Novel knowledge translation approaches may be necessary to make pre-visit educational preparation accessible and customizable for those who are less inclined to seek out educational resources on their own.

In conclusion, patients emphasized that autonomy, mobility, and quality of life are highly valued outcomes and must be integral to practice guideline development. As expected, guidance on pharmacotherapy, screening and monitoring, and fracture prevention were priorities identified to be included in osteoporosis management guidelines. The results of this national patient survey on content, outcomes, and key questions are essential for $\mathrm{OC}$ osteoporosis guidelines update process, but more importantly, this exercise emphasizes the value of patient engagement as expert-partners in the development of osteoporosis prevention and management strategies.

Acknowledgments The authors acknowledge the support of Dr. Famida Jiwa, CEO of Osteoporosis Canada and the COPN members for their participation and interest. We also thank Ms. Rachel Chepesiuk for her help with the distribution of the survey and collection of the responses. M. Larry Funnell is a patient-expert, member of COPN, and research ambassador for the Institute for Musculoskeletal Health and Arthritis of the Canadian Institutes for Health Research. SNM is a scholar of the Fonds de Recherche du Québec en Santé. WEW holds a Canada Research Chair in Bone and Muscle Development.

Funding information This study was funded in part by a Canadian Institute for Health Research planning and dissemination grant- Institute Community Support-2017-02-28 (funding reference number 152605). The conduct of the survey was supported by Osteoporosis Canada.

Compliance with ethical standards Consent to participate was assumed if the survey was completed and submitted. The study was approved by the University of Manitoba Health Research Ethics Board.

Conflicts of interest None.

Disclaimer The funding source had no role in the design of the study, analysis of the results, writing of the manuscript, or decision to submit for publication.

Open Access This article is distributed under the terms of the Creative Commons Attribution-NonCommercial 4.0 International License (http:// creativecommons.org/licenses/by-nc/4.0/), which permits any noncommercial use, distribution, and reproduction in any medium, provided you give appropriate credit to the original author(s) and the source, provide a link to the Creative Commons license, and indicate if changes were made. 


\section{References}

1. Kanis JA (2002) Diagnosis of osteoporosis and assessment of fracture risk. Lancet 359(9321):1929-1936. https://doi.org/10.1016/ S0140-6736(02)08761-5

2. Compston JE, McClung MR, Leslie WD (2019) Osteoporosis. Lancet 393(10169):364-376. https://doi.org/10.1016/s01406736(18)32112-3

3. Compston J (2005) Guidelines for the management of osteoporosis: the present and the future. Osteoporos Int 16 (0937-941X (Print): 1173-1176

4. Leslie WD, Schousboe JT (2011) A review of osteoporosis diagnosis and treatment options in new and recently updated guidelines on case finding around the world. Curr Osteoporos Rep 9(3):129-140. https://doi.org/10.1007/s11914-011-0060-5

5. Graham RMM, Wolman DM et al (2011) Clinical practice guidelines we can trust. Institute of Medicine,The National Academies Press, Washington D. C

6. Qaseem A, Forland F, Macbeth F, Ollenschläger G, Phillips S, van der Wees P, Board of Trustees of the Guidelines International Network (2012) Guidelines international network: toward international standards for clinical practice guidelines. Ann Intern Med 156(7):525-531. https://doi.org/10.7326/0003-4819-156-7201204030-00009

7. Armstrong MJ, Mullins CD, Gronseth GS, Gagliardi AR (2018) Impact of patient involvement on clinical practice guideline development: a parallel group study. Implement Sci 13(1):55. https://doi. org/10.1186/s13012-018-0745-6

8. Armstrong MJ, Bloom JA (2017) Patient involvement in guidelines is poor five years after institute of medicine standards: review of guideline methodologies. Res Involvement Engagement 3:19. https://doi.org/10.1186/s40900-017-0070-2

9. van de Bovenkamp HM, Trappenburg MJ (2009) Reconsidering patient participation in guideline development. Health Care Anal 17(3):198-216. https://doi.org/10.1007/s10728-008-0099-3

10. Légaré F, Boivin A, van der Weijden T, Pakenham C, Burgers J, Légaré J, St-Jacques S, Gagnon S (2011) Patient and public involvement in clinical practice guidelines: a knowledge synthesis of existing programs. Med Decis Mak 31(6):E45-E74. https://doi. org/10.1177/0272989X11424401

11. Armstrong MJ, Mullins CD, Gronseth GS, Gagliardi AR (2017) Recommendations for patient engagement in guideline development panels: a qualitative focus group study of guideline-naive patients. PLoS One 12(3):e0174329. https://doi.org/10.1371/ journal.pone.0174329

12. Papaioannou A, Morin S, Cheung AM, Atkinson S, Brown JP, Feldman S, Hanley DA, Hodsman A, Jamal SA, Kaiser SM, Kvern B, Siminoski K, Leslie WD, Osteoporosis SAC (2010) 2010 clinical practice guidelines for the diagnosis and management of osteoporosis in Canada: summary. Can Med Assoc J 182(17): 1864-1873. https://doi.org/10.1503/Cmaj.100771

13. Schunemann HJ, Wiercioch W, Etxeandia I, Falavigna M, Santesso N, Mustafa R, Ventresca M, Brignardello-Petersen R, Laisaar KT, Kowalski S, Baldeh T, Zhang Y, Raid U, Neumann I, Norris SL, Thornton J, Harbour R, Treweek S, Guyatt G, Alonso-Coello P, Reinap M, Brozek J, Oxman A, Akl EA (2014) Guidelines 2.0: systematic development of a comprehensive checklist for a successful guideline enterprise. Cmaj 186(3):E123-E142. https://doi.org/ 10.1503/cmaj.131237

14. Peel NM (2011) Epidemiology of falls in older age. Can J Aging 30(1):7-19. https://doi.org/10.1017/s071498081000070x

15. Zamawe FC (2015) The implication of using NVivo software in qualitative data analysis: evidence-based reflections. Malawi Med J 27(1):13-15
16. Cope DG (2014) Computer-assisted qualitative data analysis software. Oncol Nurs Forum 41(3):322-323. https://doi.org/10.1188/ 14.Onf.322-323

17. Raskind IG, Shelton RC, Comeau DL, Cooper HLF, Griffith DM, Kegler MC (2018) A review of qualitative data analysis practices in health education and health behavior research. Health Educ Behav. https://doi.org/10.1177/1090198118795019

18. Hardcastle S, Hagger MS (2011) "You can't do it on your own": experiences of a motivational interviewing intervention on physical activity and dietary behaviour. Psychol Sport Exerc 12(3):314-323. https://doi.org/10.1016/j.psychsport.2011.01.001

19. Lo AX, Brown CJ, Sawyer P, Kennedy RE, Allman RM (2014) Life-space mobility declines associated with incident falls and fractures. J Am Geriatr Soc 62(5):919-923. https://doi.org/10.1111/jgs. 12787

20. Kerr C, Bottomley C, Shingler S, Giangregorio L, de Freitas HM, Patel C, Randall S, Gold DT (2017) The importance of physical function to people with osteoporosis. Osteoporos Int 28(5):15971607. https://doi.org/10.1007/s00198-017-3911-9

21. Sale JEM, Marwah A, Naeem F, Yu W, Meadows L (2019) Evidence of patient beliefs, values, and preferences is not provided in osteoporosis clinical practice guidelines. Osteoporos Int 30(7): 1325-1337. https://doi.org/10.1007/s00198-019-04913-y

22. Utens CM, Dirksen CD, van der Weijden T, Joore MA (2016) How to integrate research evidence on patient preferences in pharmaceutical coverage decisions and clinical practice guidelines: a qualitative study among Dutch stakeholders. Health Policy 120(1):120 128. https://doi.org/10.1016/j.healthpol.2015.10.005

23. Sale JEM, Gignac MA, Hawker G, Beaton D, Frankel L, Bogoch E, Elliot-Gibson V (2016) Patients do not have a consistent understanding of high risk for future fracture: a qualitative study of patients from a post-fracture secondary prevention program. Osteoporos Int 27(1):65-73. https://doi.org/10.1007/s00198-0153214-y

24. Billington EO, Feasel AL, Kline GA (2019) At odds about the odds: women's choices to accept osteoporosis medications do not closely agree with physician-set treatment thresholds. J Gen Intern Med:1-7. https://doi.org/10.1007/s11606-019-05384-x

25. Conley RB, Adib G, Adler RA, Akesson KE, Alexander IM, Amenta KC, Blank RD, Brox WT, Carmody EE, ChapmanNovakofski K, Clarke BL, Cody KM, Cooper C, Crandall CJ, Dirschl DR, Eagen TJ, Elderkin AL, Fujita M, Greenspan SL, Halbout P, Hochberg MC, Javaid M, Jeray KJ, Kearns AE, King T, Koinis TF, Koontz JS, Kuzma M, Lindsey C, Lorentzon M, Lyritis GP, Michaud LB, Miciano A, Morin SN, Mujahid N, Napoli N, Olenginski TP, Puzas JE, Rizou S, Rosen CJ, Saag K, Thompson E, Tosi LL, Tracer H, Khosla S, Kiel D (2019) Secondary fracture prevention: consensus clinical recommendations from a multistakeholder coalition. J Bone Miner Res. https:// doi.org/10.1002/jbmr.3877

26. de Wit M, Cooper C, Tugwell P, Bere N, Kirwan J, Conaghan PG, Roberts C, Aujoulat I, Al-Daghri N, Araujo de Carvalho I, Barker M, Bedlington N, Brandi ML, Bruyere O, Burlet N, Halbout P, Hiligsmann M, Jiwa F, Kanis JA, Laslop A, Lawrence W, Pinto D, Prieto Yerro C, Rabenda V, Rizzoli R, Scholte-Voshaar M, Vlaskovska M, Reginster JY (2019) Practical guidance for engaging patients in health research, treatment guidelines and regulatory processes: results of an expert group meeting organized by the World Health Organization (WHO) and the European Society for Clinical and Economic Aspects of Osteoporosis, Osteoarthritis and Musculoskeletal Diseases (ESCEO). Aging Clin Exp Res 31:905915. https://doi.org/10.1007/s40520-019-01193-8

Publisher's note Springer Nature remains neutral with regard to jurisdictional claims in published maps and institutional affiliations. 